\title{
Apodization of Black Holes in Super-Resolution Problems
}

\author{
Evgeni Nikolaevich Terentiev ${ }^{1, ~ *}$, Irina Igorevna Farshakova ${ }^{1,2}$, \\ Nikolay Evgenyevich Shilin-Terentyev ${ }^{2}$ \\ ${ }^{1}$ M. V. Lomonosov Moscow State University, Faculty of Physics, Department Mathematical Modelling and Informatics, Moscow, Russia \\ ${ }^{2}$ EPAM Systems, Moscow, Russia
}

\author{
Email address: \\ en.teren $a$ physics.msu.ru (E. N. Terentiev), irinafarshakova $a$ gmail.com (I. I. Farshakova), \\ nikolay.terentyev@gmail.com (N. E. Shilin-Terentyev) \\ ${ }^{*}$ Corresponding author
}

\section{To cite this article:}

Evgeni Nikolaevich Terentiev, Irina Igorevna Farshakova, Nikolay Evgenyevich Shilin-Terentyev. Apodization of Black Holes in Super-Resolution Problems. American Journal of Astronomy and Astrophysics. Vol. 7, No. 3, 2019, pp. 39-47.

doi: $10.11648 /$ j.ajaa.20190703.11

Received: June 29, 2019; Accepted: July 31, 2019; Published: October 20, 2019

\begin{abstract}
Methods for identifying hidden objects are usually associated with the term apodization. The problem definitions of super-resolution must begin with the choice of the corresponding hidden object — the working discrete model of Apparatus Function (AF). With apodization such Black Holes, we will associate the mathematical invers problem of choosing working AFs with reversible $\mathrm{R}=\mathrm{O}^{-1}$ and a small inverse norm Nor $(\mathrm{R})=\|\mathrm{R}\|$. The apodization setting of super-resolution tasks, the indication of objects arise in the analysis of the Characteristics of Circumstances (CC) of the O AF models. The article provides examples of demonstration of $\mathrm{CC}$ with the choice of a discrete model of $\mathrm{AF} \mathrm{O}$ with the usual inversions $\mathrm{R}=\mathrm{O}^{-1}$ and without using a priori information about the type of solutions, as is customary in the regularization method [1]. The latter circumstance allowed us to solve the actual problem of the Super-Resolution of the image of the Black Hole Powehi shadow on low-accuracy data [16].
\end{abstract}

Keywords: Mathematical Apodization, Apparatus Function, Invers Problems, Modulation Transfer Function, Conditionality, Invertibility Indicator

\section{Introduction}

Currently, experts are taking discrete models of AF $\mathrm{O}$ in a wide range, rather arbitrarily. Data from such AF models and inversion models by mathematicians are declared incorrect $[1$, $10,11]$. From the point of view of physicists, technical specialists, such mathematical models of devices are, as a rule, not suitable for work and they need to be mathematically tuned. In this paper, we present examples when the requirement of "solving the inverse problems to look for using the a priori smoothness" leads to poor results.

In convolution operations with even reversible $\mathrm{AF} O$, $\mathrm{R}=\mathrm{O}^{-1}$ corresponds to the Modulation Transfer Function (MTF) $\mathrm{M}=\mathrm{M}(\mathrm{O}), \mathrm{M}(\mathrm{R})=1 / \mathrm{M}(\mathrm{O})$. We introduce the conditionality of the AF O DIAP $=1 /$ diap, diap $=\min |\mathrm{M}(\mathrm{O})|$, as is done in linear algebra for symmetric matrices. The most conditioned object is the Delta-Kronecker symbol DK with the MTF $\mathrm{M}(\mathrm{DK})==1$ (identically equal). For reversible AF
$\mathrm{O}$ under normalization conditions $\sum \mathrm{O}=1$, the $\mathrm{MTF}$ value at zero $\mathrm{M}(\mathrm{O})(0,0)=1$ the restriction $\operatorname{diap} \leq|\mathrm{M}(\mathrm{O})| \leq 1,1 \leq \mid \mathrm{M}$ (R) $\mid \leq$ DIAP and all values of the MTF pairs are located in the $\operatorname{diap} \leq|\mathrm{M}(\mathrm{O})|,|\mathrm{M}(\mathrm{R})| \leq \mathrm{DIAP}$.

If Nor $(R)$ is large, then we are forced to reduce Nor $(p R)$ by increasing the conditionality of $\mathrm{mD}<\mathrm{DIAP}$ (numerically, the value of $\mathrm{mD}$ decreases, and the value of $\mathrm{md}=1 / \mathrm{mD}>$ diap $=1 /$ DIAP increases) to obtain acceptable Nor $(\mathrm{pR})$. Note that Nor $(\mathrm{pR})$ is the response to noise (or the gain of the Standard Deviation (SD) $\sigma$ of white noise, $\sigma^{*}$ Nor $(\mathrm{pR})$, which determine the accuracy of solving the conversion problem in the presence of additive white noise.

\section{Basic Concepts, Problem Statements}

The main problem of the conditioned (by DIAP) choice of the $\mathrm{AF} \mathrm{pO}=\mathrm{pR}^{-1}$ is set as the minimum task (maximum precision of the solution): 
$\min _{L O}\{|| \mathrm{pR}|| \operatorname{Err}(\mathrm{pO}) \leq \mathrm{err}\}, L O=\{p O \mid[L o c, d x, m D]\}$

with the construction of the Characteristics of the Adequacy of the discrete Model (CAM) AF pO:

$$
\operatorname{CAMp}=\{\mathrm{x}=\operatorname{Nor}(\mathrm{pR}), \mathrm{y}=\operatorname{Err}(\mathrm{pO}), \mathrm{z}=\mathrm{II}(\mathrm{pR} * 0)\}
$$

Let $\mathrm{I} z=10^{-13}$ be the error of the mantissa or instrumental zero. In connection with this restriction, by mistake, consider the CAM AF version of the $\mathrm{zO}$ taking into account $\mathrm{Iz}$ the inversion of the two-dimensional MTF M (O) (in the record without indices):

$$
\begin{gathered}
\mathrm{M}(\mathrm{zR})=\left\{\begin{array}{c}
\frac{1}{M(0)}, \text { for } \operatorname{all}|\mathrm{M}(\mathrm{O})|>I z \\
M(O)
\end{array}\right. \\
\mathrm{CAMz}=\{\mathrm{x}=\operatorname{Nor}(\mathrm{zR}), \mathrm{y}=\operatorname{Err}(\mathrm{zO}), \mathrm{z}=\mathrm{II}(\mathrm{zR} * 0)\}
\end{gathered}
$$

We introduce a numerical function - the Invertibility Indicator in order to know if we decided whether the AF O invertibility problem or not. By definition: II $\left(z R^{*} \mathrm{O}\right)=\mathrm{zR} * \mathrm{O}$ $(0,0)$. If the value of the invertibility indicator II $(z R * O)=1$, then the inversion is $z R=R=\mathrm{O}^{-1}$. If II $\left(z R^{*} \mathrm{O}\right)<1$, then we have an incomplete $\mathrm{Iz}$ inversion of $\mathrm{AF} \mathrm{O}$ in $\mathrm{zR}$. Similarly, for conditional tunable reversibility II $\left(\mathrm{pR}^{*} \mathrm{O}\right)$ for estimating the value of reversibility with a given value.

Of course, the apodization of $\mathrm{AF} \mathrm{O}$ is preceded by the construction of $\mathrm{CC}$ discrete $\mathrm{AF} \mathrm{zO}$ models for fixed continuous AF parO:

$$
\mathrm{CC}=\{\mathrm{x}=\mathrm{Dx}, \mathrm{y}=\operatorname{Nor}(\mathrm{zR}), \mathrm{z}=\mathrm{Loc}\}
$$

In CC (5), Loc is an array of lengths of the definition areas of continuous parO AF, and Dx is an array of steps dx of digitizing $\mathrm{AF}$ parO. In the examples $\mathrm{XO} \mathrm{AF} z \mathrm{z}$, the complete invertibility is noted with an asterisk * inside the symbol $\mathrm{O}$, see Figures 2-3.

\section{Super-Resolution Function, SR Values, CC, Apodization Problems}

\subsection{Definition of Super-Resolution Function}

Suppose there is a normalization for AF O:

$$
\sum \mathrm{O}=\sum_{\mathrm{i}, \mathrm{j}=1}^{\mathrm{Loc}+1} \mathrm{O}_{\mathrm{ij}}=1,
$$

then the $\operatorname{MTF} \operatorname{M}(O)(0,0)=1$, in this case we will estimate the tunable Super-Resolution by value

$$
\begin{gathered}
\mathrm{SR}=\mathrm{BW} / \sum_{\mathrm{i}, \mathrm{j}=1}^{\mathrm{Loc}+1} \mathrm{M}_{\mathrm{ij}}(\mathrm{pO}) \leq \sum_{\mathrm{i}, \mathrm{j}=1}^{\mathrm{Loc}+1} 1 / \sum_{\mathrm{i}, \mathrm{j}=1}^{\mathrm{Loc}+1} \mathrm{M}_{\mathrm{ij}}(\mathrm{pO}) \geq 1, \\
\mathrm{BW}=\sum_{\mathrm{i}, \mathrm{j}=1}^{\mathrm{Loc}+1} \mathrm{M}_{\mathrm{ij}}(\mathrm{pR}) \mathrm{M}_{\mathrm{ij}}(\mathrm{O})
\end{gathered}
$$

where $\mathrm{BW}(8)$ is the BandWidth. If $\mathrm{AF} \mathrm{O}=\mathrm{D}_{\mathrm{K}}$, then $\mathrm{SR}=1$. It is clear that the problem of super-resolution arises for cases when $\mathrm{AF} \mathrm{O} \neq \mathrm{D}_{\mathrm{K}}$.

The super-resolution expression SR is the function of the parameters [Loc, dx, mD] (1). Examples of SR functions for $\mathrm{AF} \mathrm{O}_{\mathrm{G}}$ images of a Martian stone in $\mathrm{G}$ color are given in figure 1. Image of Martian stone is presented in the text below.

It is desirable that the value of super-resolution SR does not depend on the size of images and large Loc.

\subsection{About Values of SR Function}

In reversible cases, SR (7) as a function of three parameters [Loc, $\mathrm{dx}, \mathrm{mD}$ ] is a function with saturation by two parameters: the length of the domain of definition of Loc and the conditionality of $\mathrm{mD}$. In the discrete case $\mathrm{dx}=1$, this is seen in Figure 1 on the left (a). In the continuous case of $d x=1 / 4$, this is evident in Figure 1 on the right (b) for large values of DIAP in the case of Iz inversions.

Moreover, since the image size, as a rule, is larger than the size of the Loc definition domain, the value (with saturation) of the conditioned super-resolution SR does not depend on the image size.

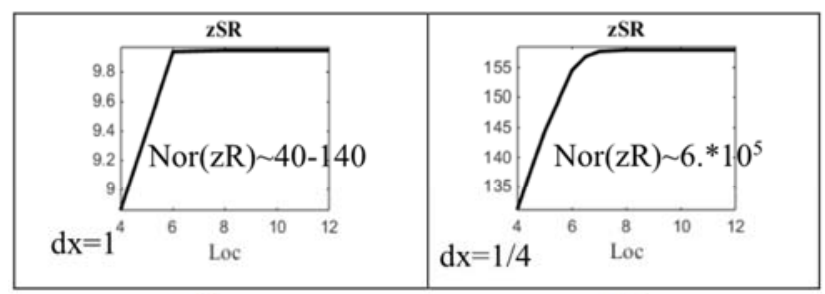

(a)

(b)

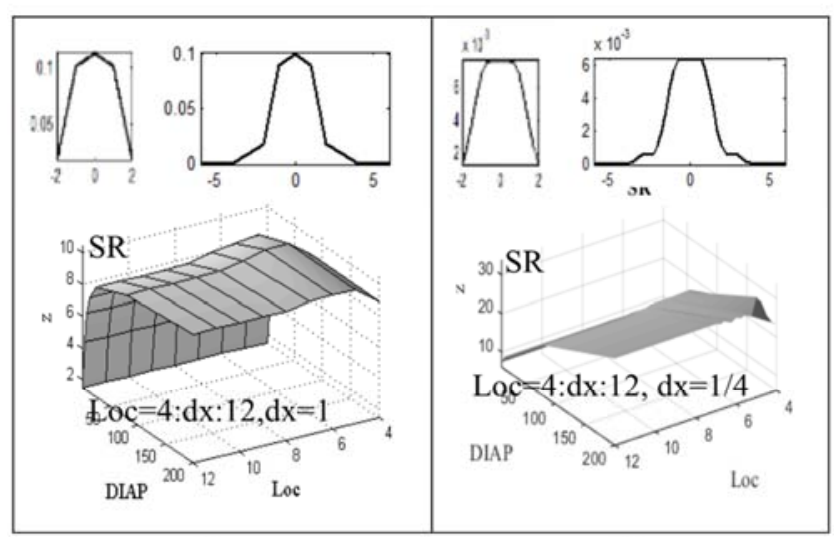

(c)

(d)

Figure 1. An example of SR functions in discrete $d x=1$ and continuous (with interpolation) case with $d x=1 / 4$.

$\mathrm{SR}$ (6) for Martian images with $\mathrm{AF} \mathrm{O}_{\mathrm{G}}$ is a function with saturation. On the initial grid with $d x=1 \mathrm{zSR} \sim 10$ in saturation with the response to the noise Nor (zR) 40-140. For interpolated AF $\mathrm{O}$ and accordingly images we can theoretically get $\mathrm{zSR} \sim 160$, with a huge response to noise $\sim$ Nor (zR) $6^{*} 10^{5}$. Separately, the graphs of the SR functions are presented, in the discrete and continuous case, if the $\mathrm{SR}$ is tuned to the DIAP conditionality, see Figure 1. 


\section{3. $\mathrm{AF} \mathrm{pO}$ and $\mathrm{zO}$ Errors}

The continuous parametric AF parO model, we associate the discrete $\mathrm{AF} \mathrm{O}$ with the calculation (direct transformation) of the MTF M (O). If we build an inverse AF according to $M$ $(\mathrm{O})$, then we get the result of the $\mathrm{AF} \mathrm{zO}$ with the instrumental (mantissa) error Iz. The conditional adjustment according to DIAP is that small values of $\mathrm{M}=\mathrm{M}(\mathrm{O})$ move away from Iz by values $\mathrm{md}>\operatorname{diap}=\min |\mathrm{M}(\mathrm{O})|=1 / \mathrm{DIAP}$ with the production of MTF pM. We realize the inverse transformation from MTF $\mathrm{pM}$ to $\mathrm{AF}$ pO [3]. Error Err (pO) we estimated [7-9] by the formula:

Err $(\mathrm{pO})=\mathrm{SD}(\mathrm{O}-\mathrm{pO}) / \max (\mathrm{O})$, where

$$
\mathrm{SD}(0-\mathrm{p} 0)=\operatorname{sqrt}\left(\sum(0-\mathrm{pO})^{2} /(\operatorname{Loc}+1)^{2}-1\right)
$$

Err (pO) (9) errors are present in CAM AF pO (2).

\subsection{Examples of $C \mathrm{CAF} O$}

Until now, inverse problems of mathematics are characterized by everyday terms such as the Ill-posed problem, bad ones, etc. In this section, we will introduce Circumstances as a mathematical object. The purpose of this introduction is that there may be Good Circumstances (GC) in the CC, which will prompt us the correct formulation of the problem.

As a first example, we will take the digitization of the standard Gaussoid - GAU parO $=\exp \left(-\mathrm{x}^{2} / 2\right)$, turning them into discrete models AF O with normalization $\sum \mathrm{O}=1$ (6).

\section{$\operatorname{Loc}=4: \mathrm{dx}: 10$}

GAU parO, $\sigma=1$

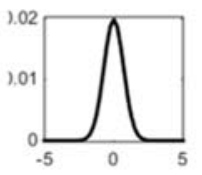

$\mathrm{Dx}=\left[\begin{array}{lllllll}0.2 & 0.35 & 0.43 & 0.5 & 0.6 & 0.7 & 1\end{array}\right]$
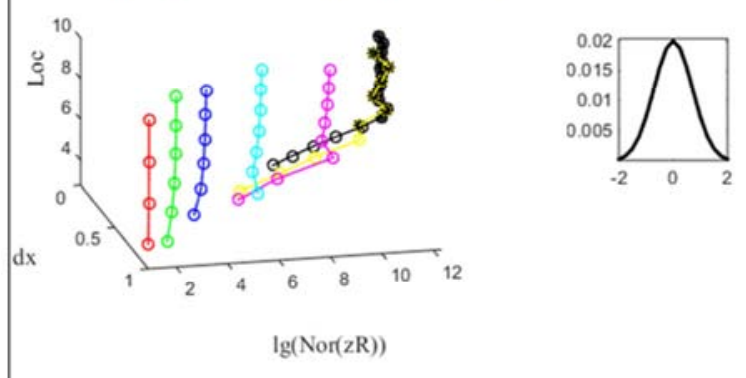

(a)

Figure 2. CC AF parO is Gaussoid, an asterisk * indicates irreversible options.

In coarse digitization grids, there is an inversion of $\mathrm{AF} O$ with small norms Nor (R). In grids with $d x=0.2$ and 0.35 for
Loc $=6$, we lose the reversibility, see asterisks *. The loss of reversibility with small $\mathrm{dx}$ and beyond Loc $=6$ occurs according to the " $3 \sigma$ " rule for the density of the normal distribution. GC with small Nor (R) are implemented with large steps of digitizing $\mathrm{dx}$.

Loc $=8: \mathrm{dx}: 26$

REC parO, ST=5

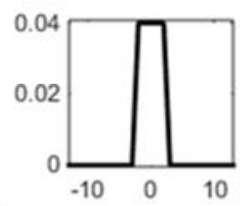

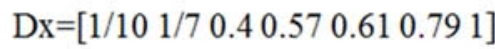

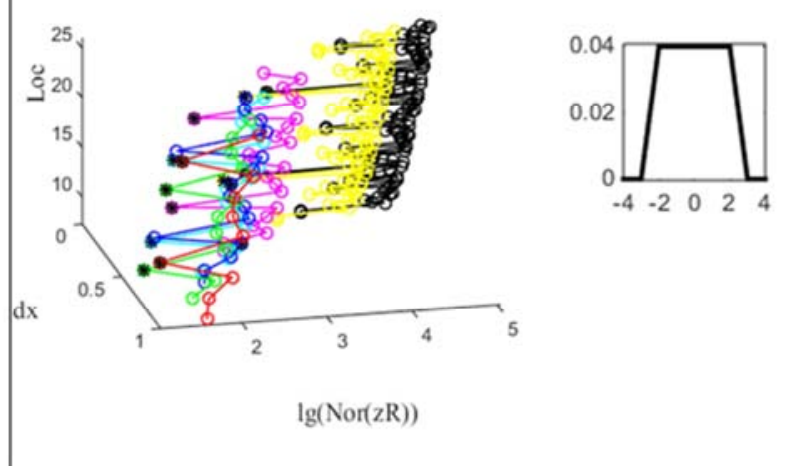

(a)

Figure 3. $X O A F$ parO Rectangle with $S T=5$.

For small $\mathrm{dx}$ from $\mathrm{Dx}$, reversibility takes place with large Nor (R). With coarse meshes, it turned out that difficult situations are taking place. Reversible working options must be sought.

\subsection{Apodization Problems 5/25\&5/26}

In foreign scientific literature before the regularization method A. Tikhonov. [1] for solving inverse problems was proposed Windows method in the frequency domain. In the simplest versions of the Windows method, the AF O was simply inverted within the low frequencies in Windows, and behind Windows, the high frequencies were reset. However, it turns out not so simple.

In the example below, we consider the presence of MTF values $\mathrm{M}(\mathrm{O})$ for $\mathrm{AF} O$ falling near the instrumental error Iz depending on the length of the definition domain $\mathrm{N}$. These are special cases of the already analyzed $\mathrm{CC}$ example with REC parO $\mathrm{ST}=5, \mathrm{dx}=1$. An example is characteristic of apodization: with $\mathrm{N}=25 \mathrm{AF}$, $\mathrm{O}$ with $\mathrm{ST}=5$ is not reversible. If one point is added to the domain of definition, then AF $\mathrm{O}$ becomes reversible, because these small values of $\mathrm{M}(\mathrm{O})$ become large and leave the vicinity of Iz errors, see Figure 4. 


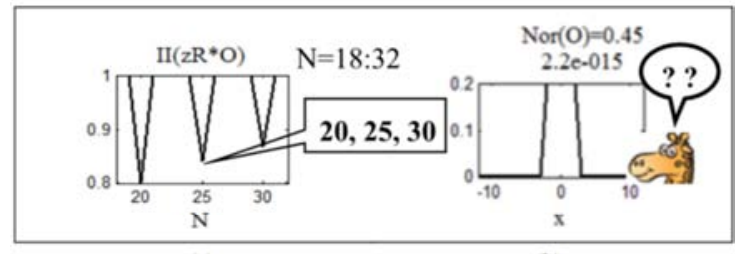

(a)

(b)

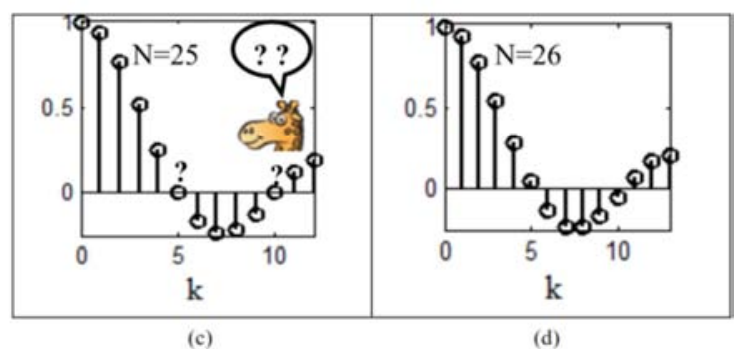

Figure 4. Demonstration of the cause of reversibility and irreversibility in the halves of the MTF $M(O)$ for $A F O S T=5$ with $N=25$ and $N=26$.

For a set of lengths of definition domains $\mathrm{N}=18: 32$, this situation is realized three times with $\mathrm{N}=20,25$, and 30 . Reversible cases differ in the value of Nor (R). In Figure 4 we have identified an irreversible case with a sign of astonished

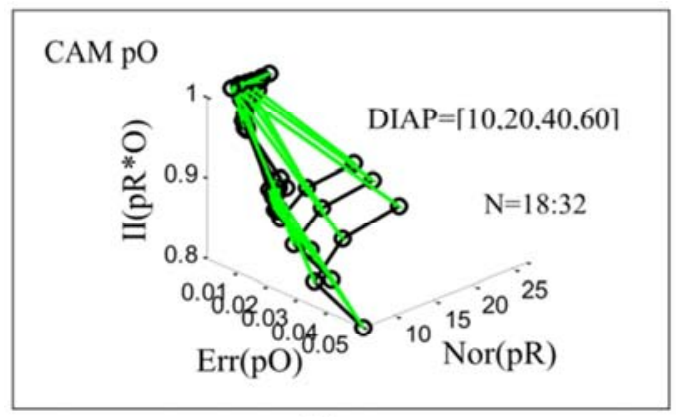

(a) giraffes.

When interpreting the solution of the super-resolution problem, we must give the value of the inverse error $\operatorname{Err}(\mathrm{pO})$ and for the regularization method $\operatorname{Err}(\mathrm{aO}), \mathrm{aO}=\mathrm{aR}^{-1}$.

For the regularization method $[1,10,11]$, just in cases of irreversibility with $\mathrm{N}=20,25$, and 30 , these relative inverse errors turned out to be of the order of $\sim 2 * 10^{13}$, i.e. we cannot interpret the solution of the super-resolution problem under these $\mathrm{N}$, see the sign of the giraffes.

Note that there are areas in CAM pO and CAM aO where everything is good: small Nor (pR), Nor (aR), reversible cases along the $\mathrm{z}$ axis are distinguished by indicators of reversibility $\mathrm{z}=\mathrm{II}(\mathrm{pR} * \mathrm{O})=1, \mathrm{z}=\mathrm{II}(\mathrm{aR} * \mathrm{O})=1$, see Figure 5 .

Note that the variation principles with the Lagrange functions, the Tikhonov functional, the Euler equation to the choice of discrete models of AF O are not directly related, and the old Windows method is too rough [1-5].

From the point of view of apodization: it is necessary to choose working models with minor (pR). The absence of the requirement of a priori smoothness of solutions (as in the regularization method [1]) allows us to see point objects in over-resolved images [12-15].

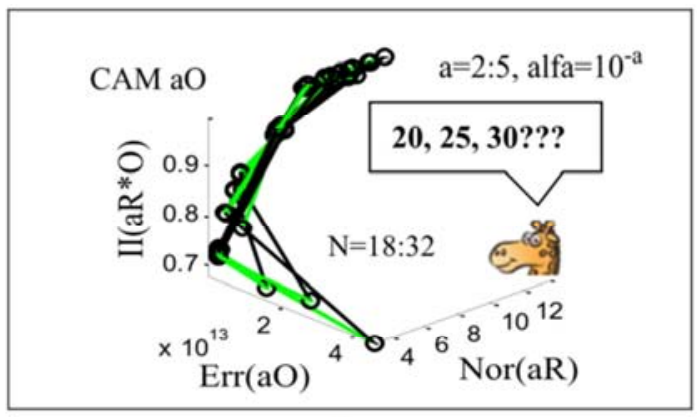

(b)

Figure 5. Examples of CAM $\mathrm{PO}$ and CAM aO with a demonstration of the reverse errors Err (pO) and Err (aO) for the regularization method.

In the next two sections, more complex cases are considered with the addition of a beam to the design of a multibeams $\mathrm{AF}$ $\mathrm{O}$ [7-9], with the CAM option in a grid with $\mathrm{dx}=1$ and $\mathrm{N}=110$ : 120 - the dimensions of the image definition areas.

\section{Multi-Beams AF O}

\subsection{Reversible Case of Five-beam $A F \mathrm{O}=5 \mathrm{o}$ with a Minimum Nor (R)}

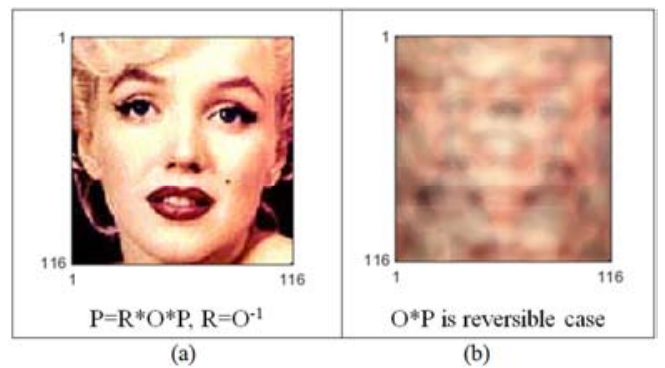

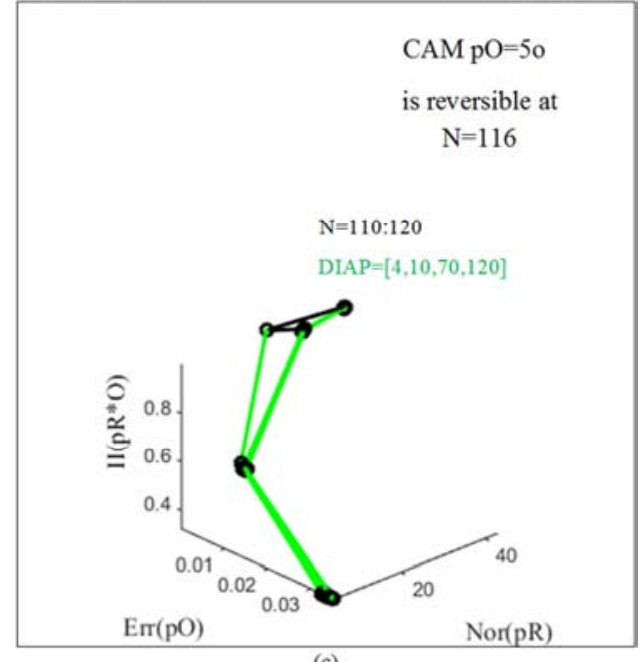

Figure 6. When $N=116$, the distortion of the $A F O=50$ is reversible, II $(z R * O)=1$.

The 5-ray AF $\mathrm{O}=50$ is reversible with a small norm of Nor 
(R) 24.2 at $\mathrm{N}=116$. If $\mathrm{N}$ is different, then Nor (pR) can be adjusted according to $\operatorname{DIAP}(1,2)$ conditionality with $\operatorname{Err}(\mathrm{pO})$ error.

Figure 7 presents data on the properties of $\mathrm{AF} O=5 \mathrm{o}$. Type inverse $\mathrm{R}=\mathrm{O}^{-1}$, the result $\mathrm{R}^{*} \mathrm{O}=\mathrm{DK}$ - Delta-Kronecker symbol.

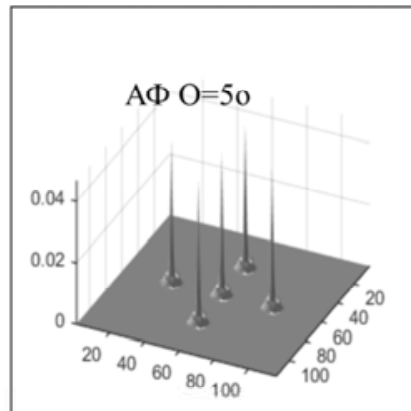

(a)

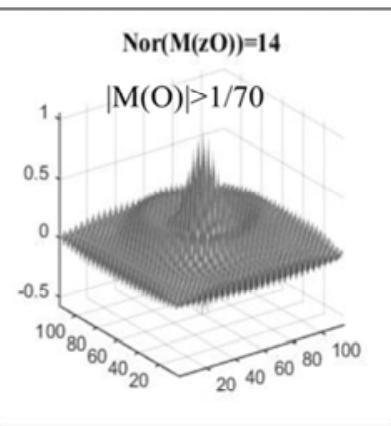

(b)

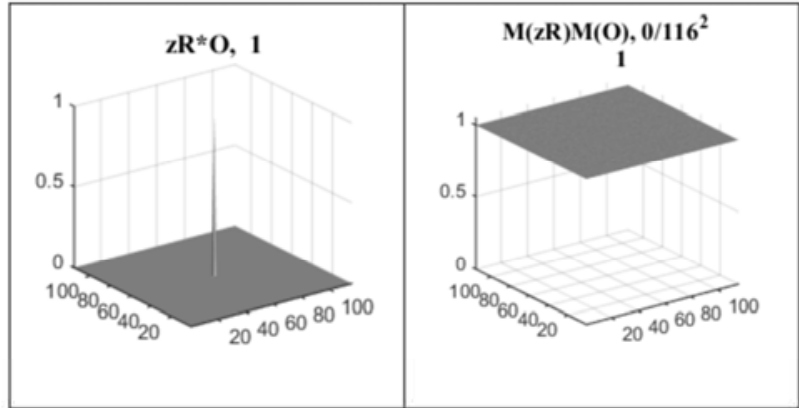

(e)

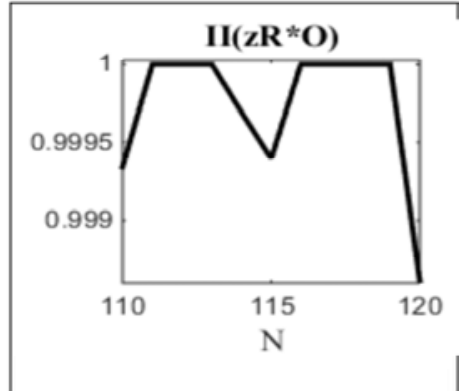

(g)
(R) $\mathrm{M}(\mathrm{O})==1$. Note that the through zero $|\mathrm{M}(\mathrm{O})|>1 / 70$, which follows from the form $\mid \mathrm{M}$ (R) $\mid<70$. Next to the domain size $\mathrm{N}=115$, we have the irreversible case II $\left(z R^{*} \mathrm{O}\right)=0.9995$.

(c)

(d)

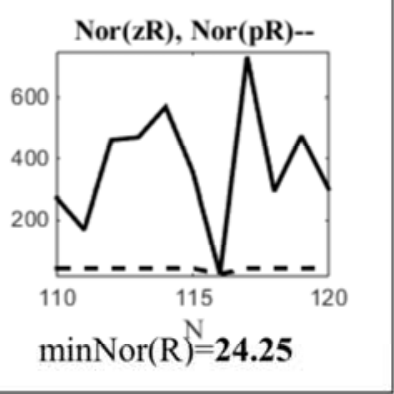

(h) MTF $\mathrm{M}(\mathrm{O})$ is an oscillating function that does not pass

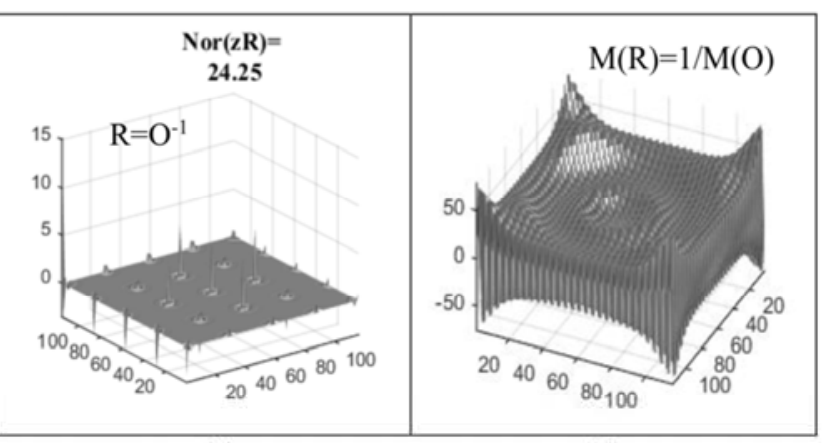

Figure 7. $A F O=50$ with $M T F|M(O)|>1 / 70$, minor $(R) \sim 24.25, N=116$.

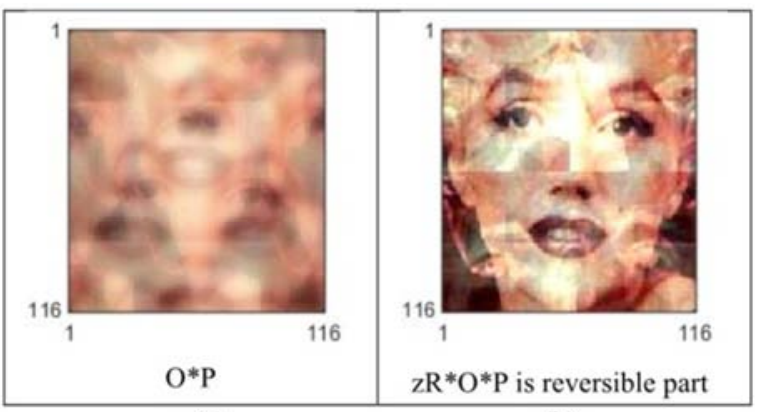

(a)

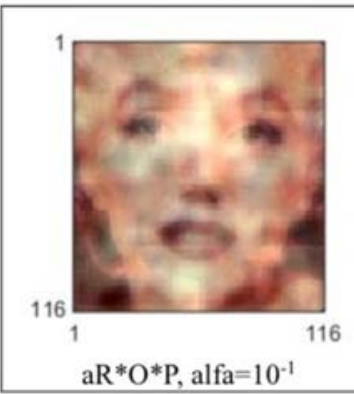

(c) (b)

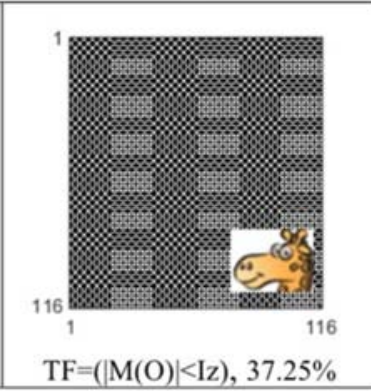

(d)
Figure 8. At $N=116$, the distortion of the $A F O=4 o$ is not reversible: II $(z R * O)=0.625$

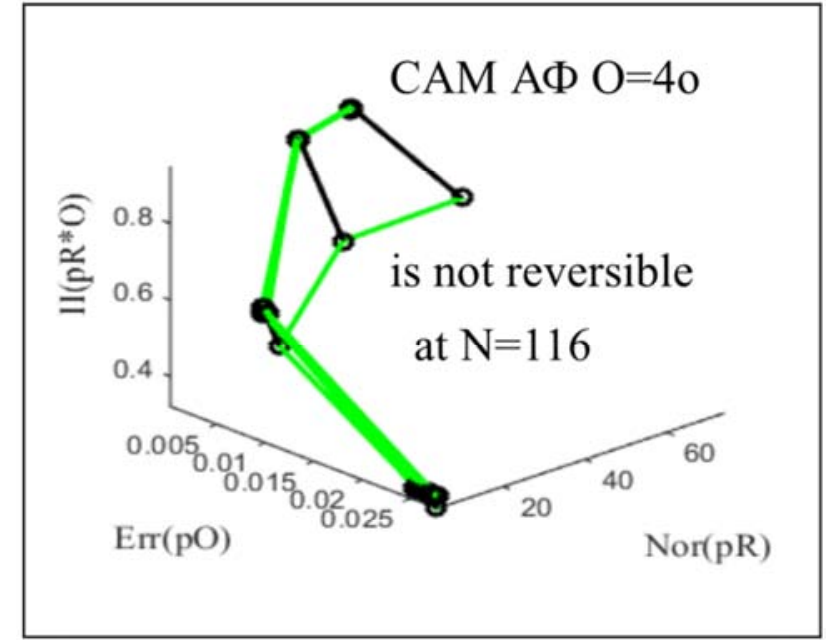

(a)

Figure 9. When $N=116$, the distortion of the $A F O=4 \mathrm{o}$ is not reversible, II $\left(z R^{*} O<1\right.$.

\subsection{The Case of Four-beam $A F \mathrm{O}=40$ with Irreversible $O$ and Minimal Nor $(z R)$}

If in the 5-ray system we remove the central ray, then we get a completely different irreversible mathematics $[8,9]$.

The reversibility indicator II $(\mathrm{zR} * \mathrm{O})=0.625$ indicates that 
many values of the MTF M (O) oscillating M $(\mathrm{O})$ are in the region of the instrumental error Iz. This means the irretrievable loss of information about the image. In Figure 7 a separate picture represents a logical (binary $0-1$ ) image $\mathrm{TF}=$ $(\mathrm{M}(\mathrm{O})<\mathrm{Iz})$.
5046 points or $37.25 \%$ of the values of the MTF M (O) fell into the instrumental error. A giraffe marks this situation. We draw attention to the reversible part of $<<\mathrm{zR} * \mathrm{O} * \mathrm{P}>>$ presented in Figure 8 (b).

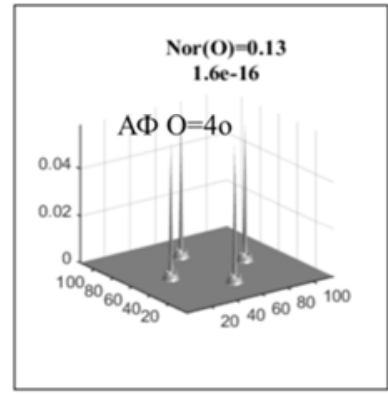

(a)

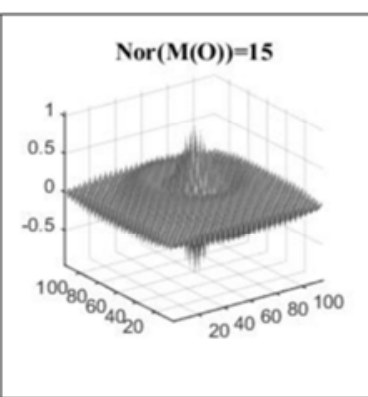

(b)

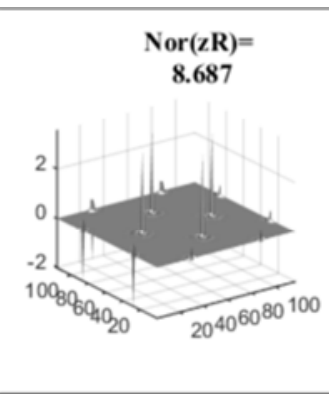

(c)

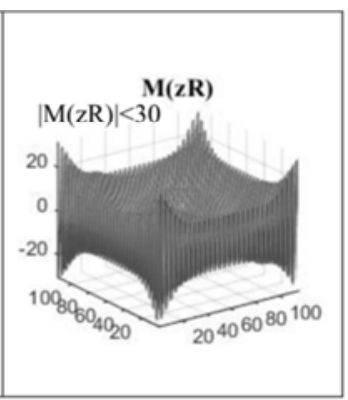

(d)

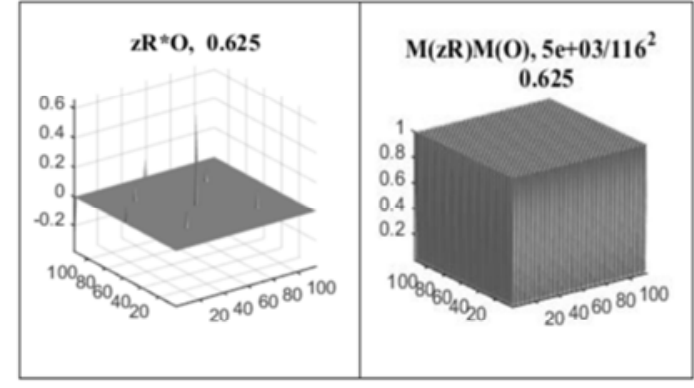

(e)

(f)

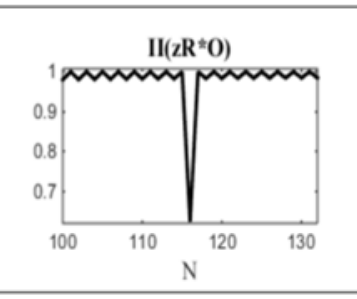

(g)

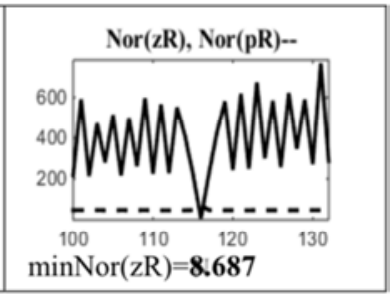

(h)

Figure 10. There is no reversibility of $A F O=4 o: I I(z R * O)=0.625$.

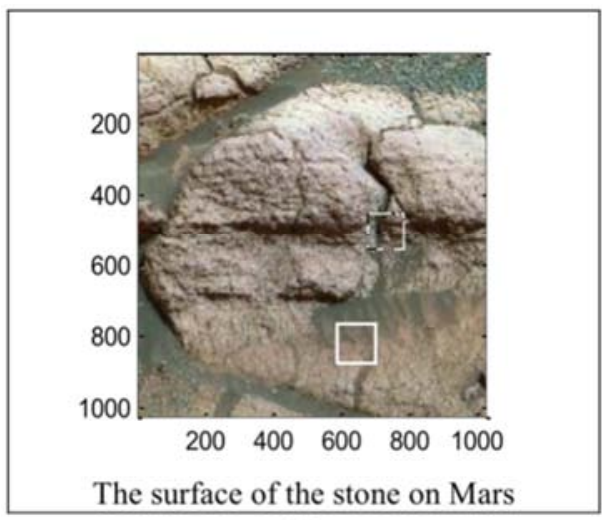

(a)

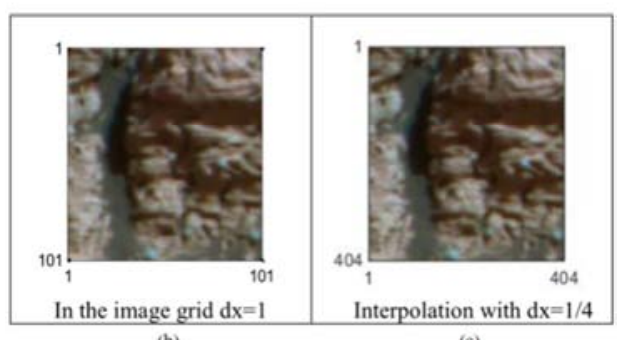

(b)

Figure 11. On the image of a stone on Mars, there are two areas, on the bottom - sand in the form of dunes.

Examples of the last sections suggest that a priori information about the type of solutions only worsens the result for solutions with a minimum norm in a reversible and irreversible case. All this was done according to $<<$ CAM pO $>>$ and according to the data of the reversibility indicator: $<<$ if II $(\mathrm{zR} * \mathrm{O})=1$, then $\mathrm{zR}=\mathrm{R}=\mathrm{O}^{-1}>>$, associated with the instrumental error Iz.

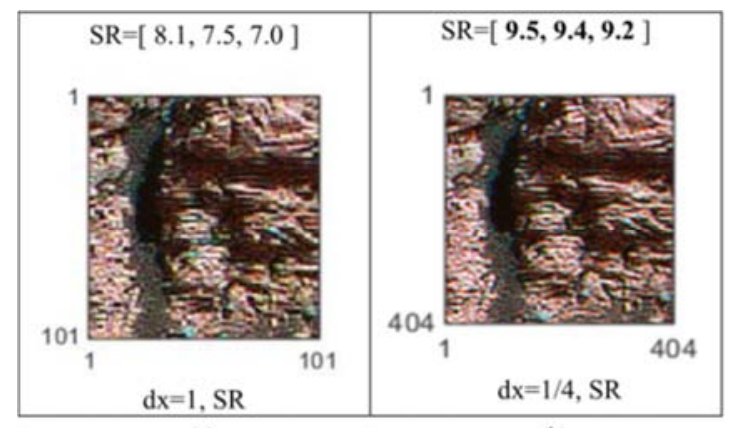

(a)

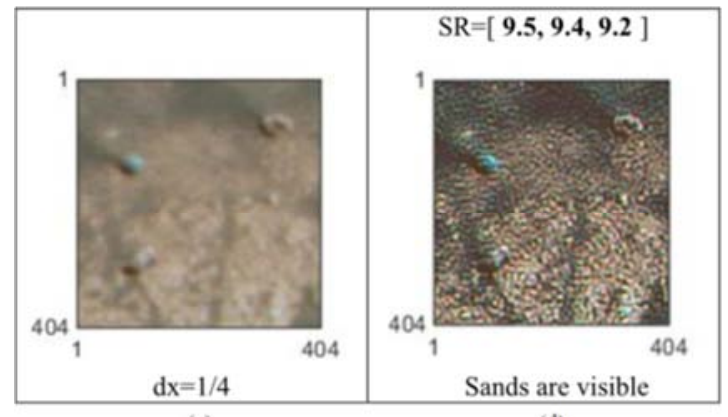

(c)

Figure 12. Supe - Rresolution during interpolation with $d x=1 / 4 A F$ and the original image $\sim 20 \%$ higher than on the original grid with $d x=1$. 


\section{The Resolution of Objects (Sand) Is Less than a Pixel}

Image system developers are usually reinsured and choose a pixel size smaller than the effective size (spot) of AF O. The question arises: Can we see objects smaller than a pixel in such an image registration system?

The idea of the solution is that AF $\mathrm{O}$ and the image are interpolated and, according to the CAM pO, we plan Super Resolution with a choice of conditionality $\mathrm{mD}=1 / 15$. In the continuous-interpolated case, the SR values turned out to be $15-20 \%$ higher than in the discrete case, which is confirmed by the resulting images, see Figure 12 .

\section{Super-Resolution of the Black Hole Powehi Shadow}

The image of the shadow of the Black Hole Powehi was recorded by the Event Horizon Telescope [16] in three radio bands $\{R, G, B\}$ consisting of 8 receiving synchronized antennas with a single antenna pattern or $A F \mathrm{O}_{\mathrm{T}}=\left\{\mathrm{O}_{\mathrm{R}}, \mathrm{O}_{\mathrm{G}}\right.$, $\left.\mathrm{O}_{\mathrm{B}}\right\}$, Loc $=60$, see Figure 13 .

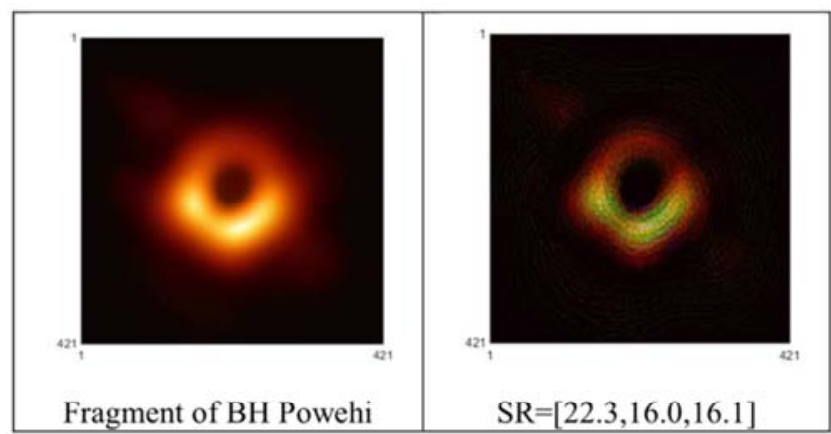

(a)

(b)

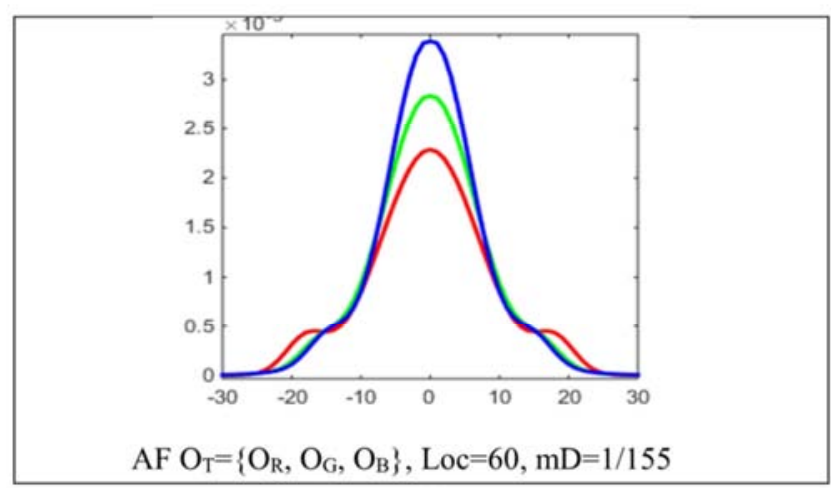

(c)

Figure 13. Fragment of shadow BH Powehi, graphs of $A F O_{T}$ through three channels $R, G, B$ with $L o c=60$.

We assumed that individual points on channel B are stars falling in BH. The apodization search for a solution without the use of a priori information about the smoothness of solutions helped us to resolve individual stars.

\subsection{Discussion About SR BH Powehi}

"To pull out - to assess the AF $\mathrm{O}_{\mathrm{T}}$ " solution of the problem of Super Resolution BH helped us just these stars falling in $\mathrm{BH}$, see Figure 14 (b).

Note that the task Super Resolution $\mathrm{BH}$ was solved according to low accuracy data - the first byte image (in 104kb and just for publication in press) from the Internet.

\subsection{Future SR BH Powehi}

Now we have high precision data (grid is 100 times denser and brightness in two bytes and one image is in $183 \mathrm{MB}$ ), on which results are expected with Hyper-Resolution (HR), possibly exceeding this by dozens times SR. We want to present this unique result with HR BH Powehi at the Moscow Russian-Chinese symposium in October.

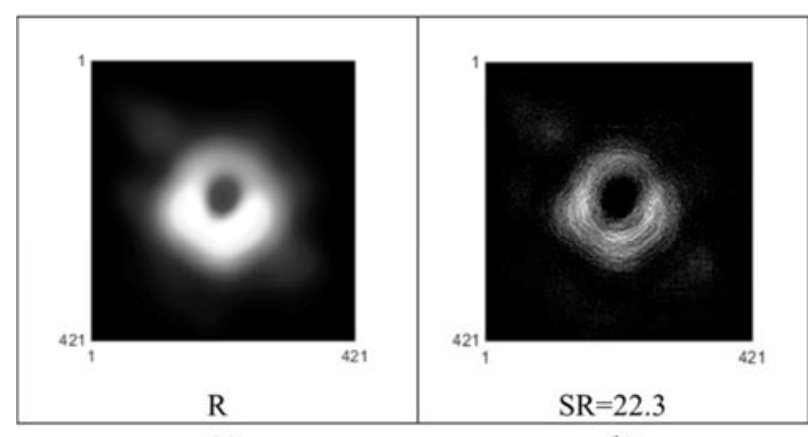

(a)

(b)

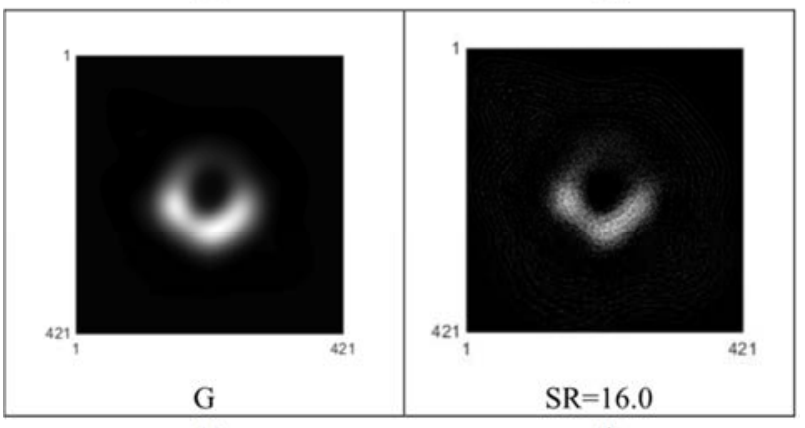

(c)

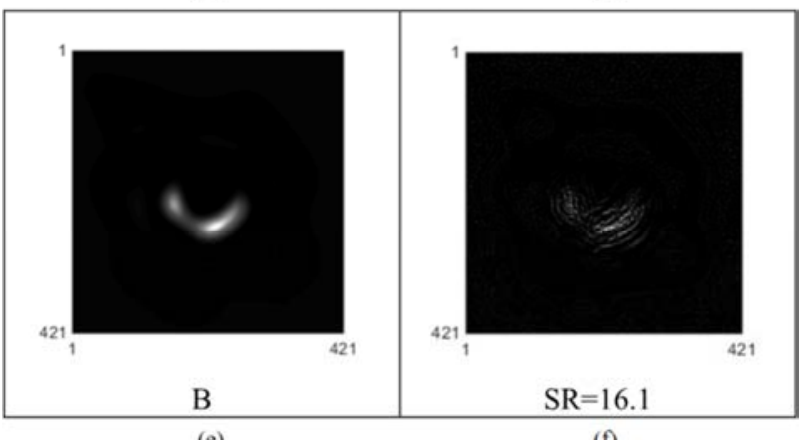

(e)

(f)

Figure 14. Comparison of the original and Super Resolved images for each radio band $R, G, B$.

With the title of the article, we emphasize that the methods for solving inverse problems with unknown $\mathrm{AF} O$ (or compensating for distortions of a complex of telescopes with an unknown antenna pattern) are BH in mathematics. 
We hope that we will be able to obtain a much higher Super Resolution on high-precision data using the apodization method [12-15], to refine the antenna pattern.

Since the volume of high-precision data is more than 100-200 times larger, we plan to center the fragments of the resulting images on the selected stars in channel $\mathrm{B}$.

\section{Conclusions and Plans}

$\mathrm{CC}$ show that in complex $\mathrm{AF} \mathrm{O}=5 \mathrm{o}$, the decision on the distortion compensation is obtained by the usual treatment with a small minNor $(\mathrm{R}) \sim 24.2$, a priori information about the type of solutions [1] is not needed. In the irreversible case of AF $\mathrm{O}=4 \mathrm{o}$, a priori information is not needed because the decision error increases and the resolution decreases due to it.

This approach can be used in simulating the inconsistency of the Event Horizon Telescope [16] when assembling the resulting images and solving subsequent SR problems.

Estimated imbalance of 8 radio telescopes, in the radio $\mathrm{R}$ range was minimal, therefore a higher $\mathrm{SR}=22.27$ was obtained.

The plans of the task of increasing the resolution of $3 \mathrm{D}$ objects in CT and MRT analysis of tumors, ulcers, etc. in medical diagnostics using field theory operations and their modifications.

Now in astronomy, many areas of possible applications. These are all tasks from X-ray telescopes, radio telescopes. There are plans to solve the super-resolution problem in the study of the mechanism of nucleation and growth of binary stars around the binary protostar L1551 NE, located in the constellation Taurus.

At the ALMA Observatory in Chile, an image of a protoplanetary disk with emerging planets. Now the attention of astronomers is riveted on the Black Hole Sagittarius A* in the center of the Milky Way. The detailing of the complex interrelationship between the $\mathrm{BH}$ and its galactic neighborhood for astronomers is of great interest.

\section{Acknowledgements}

The work was done on its own initiative, without grant support.

\section{References}

[1] A. N. Tikhonov, M. V. Ufimtsev "Statistical processing of experimental results", Moscow University Press, 1988 (in Russian).

[2] Evgeni N. Terentiev, Nikolay E. Terentiev, M. V. Lomonosov Moscow State Univ. (Russia), Applications of pointed ultra-resolution method in colour imaging, published in Proceedings Volume 5817: Visual Information Processing XIV, May 2005, Available on the SPIE Digital Library.

[3] Evgeni N. Terentiev, M. V. Lomonosov Moscow State Univ. (Russia); Nikolai E. Terentiev, XSIA (Russia); Fedor V. Shugaev, M. V. Lomonosov Moscow State Univ. (Russia), Inside the ultra-resolution method, published in Proceedings Volume 5574: Remote Sensing for Environmental Monitoring,
GIS Applications, and Geology IV, October 2004, Available on the SPIE Digital Library.

[4] Evgeni N. Terentiev, M. V. Lomonosov Moscow State Univ. (Russia); N. E. Terentiev, Quest Software Inc. (Russia), Characterization of ultra-resolution method, published in Proceedings Volume 6246: Visual Information Processing XV, May 2006 - View Abstract, Available on the SPIE Digital Library.

[5] Evgeni N. Terentiev, M. V. Lomonosov Moscow State Univ. (Russia); Nikolay E. Terentiev, Quest Software Inc. (Russia), Ultra-resolution and indication of objects, published in Proceedings Volume 6211: Passive Millimeter-Wave Imaging Technology IX, May 2006 View Abstract, Available on the SPIE Digital Library.

[6] Evgeni N. Terentiev, Nikolai E. Terentiev, M. V. Lomonosov Moscow State Univ. (Russia), Superresolution when PSF is indefinite, published in Proceedings Volume 4388: Visual Information Processing X, August 2001, Available on the SPIE Digital Library.

[7] Terentiev E. N., Terentyev N. E. CHARACTERISTICS OF ADEQUACY OF MODELS OF MEASURING COMPUTING SYSTEMS, Proceedings of the XIX International Forum on problems of science, technology and education, p. 95-97 (2015) (in Russian).

[8] Terentyev E. N., Terentyev N. E. PROBLEMS OF MULTI-BEAM MEASURING - COMPUTING SYSTEMS, Proceedings of the XIX International Forum on problems of science, technology and education, p. 94-95 (2015) (in Russian).

[9] Terentiev E. N., Terentyev N. E. ADEQUATE SETTINGS OF RAY CREST IN RADAR TECHNOLOGIES, Proceedings of the XIX International Forum on the problems of science, technology and education, p. 76-78, (2015) (in Russian).

[10] E. N. Terentiev, N. E. Terentyev MATHEMATICAL PRINCIPLES OF SETTING MEASURING-COMPUTING SYSTEMS AND REGULARIZATION, NOTES OF THE RAS, PHYSICAL SERIES, 2015, Volume 79, No. 12, p. 1633-1637 (in Russian).

[11] I Terentiev, E. N. and Terentiev, N. E. //ISSN 1062-8738, Bulletin of the Russian Academy of Science. Physics, 2015, Vol. 79, No 12, pp. 1427-1431, DOI $10.3103 / \mathrm{S} 1062873815120229$.

[12] E. N. Terentyev, N. E. Terentyev, Yu. A. Pirogov, I. I. Farshakova, Physical Principles for Setting Apparatus Functions of Measuring Instruments, SCIENTIFIC NOTES OF THE PHYSICAL FACULTY OF MOSCOW UNIVERSITY, 9 pp., No. 6, 1761005 (2017) (in Russian).

[13] E. N. Terentyev, N. E. Shilin-Terentyev Conditional super-resolution with classifiers, SCIENTIFIC NOTES OF THE PHYSICAL FACULTY OF THE MOSCOW UNIVERSITY, No. 5 1850306, p. 1-9 (2018) (in Russian).

[14] Terentiev E. N., Terentiev N. E., Farshakova I. I., PMMEEP 2017, Physical and Mathematical Modeling of Earth and Environment Processes, 19 Chapter, Principles of Controlling the Apparatus Function for Achieving Super-Resolution in images, pp. 171-182, Number of pages 382, Springer International Publishing, DOI: 10.1007/978-3-319-77788-7_19C. 
[15] E. N. Terentiev, N. E. Shilin-Terentiev, Physical and Mathematical Modeling of Earth and Environment Processes (2018), Classifiers in Super-Resolution Problems, pp. 441-455, Springer Proceedings in Earth and Environmental Sciences. Springer, Cham, doi.org/10.1007/978-3-030-11533-3_44.
[16] The Event Horizon Telescope Collaboration, First M87 Event Horizon Telescope Results. I. The Shadow of the Supermassive Black Hole, The Astrophysical Journal Letters, 875: L1 (17pp), 2019 April 10, https://doi.org/10.3847/2041-8213/ab0ec7. 